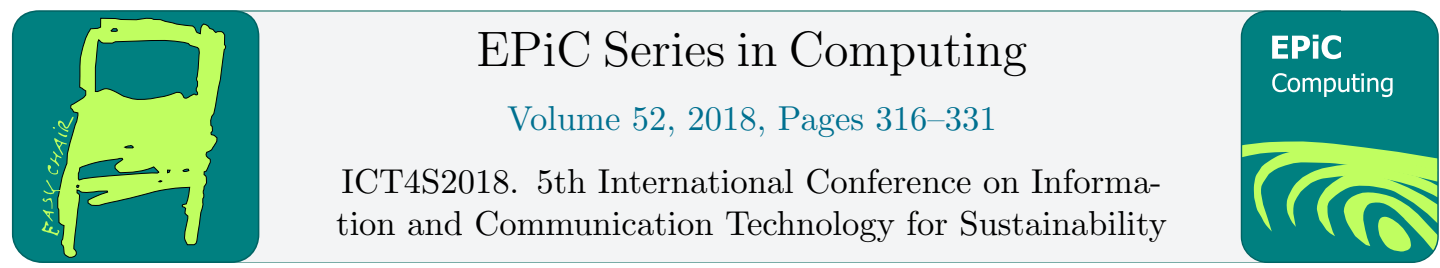

\title{
"Sustainability... it's just not important." The Challenges of Academic Engagement with Diverse Stakeholders
}

\author{
Christian Remy ${ }^{1}$, Oliver Bates ${ }^{2}$, Vanessa Thomas ${ }^{3}$, and Matthew Broadbent ${ }^{2}$ \\ ${ }^{1}$ Centre for Digital Creativity, Aarhus University, DK, remy@cc.au.dk \\ 2 School of Computing and Communication, Lancaster University, UK, \\ \{o.bates, a.friday\}@lancaster.ac.uk \\ ${ }^{3}$ Department of Computer Science, Aarhus University, DK, vthomas@cs.au.dk
}

\begin{abstract}
Research at the intersection of sustainability and computing often engages with external stakeholders, and those engagements can determine success and impact of the sustainable goals as well as the entire research. In this paper, we reflect on various stakeholder engagements to derive lessons for the ICT4S community that seeks to broaden its sustainable impact beyond their own field. We briefly describe our experiences with case studies from each of the individual author's research, and then synthesize across those experiences to reflect on similarities and differences. The resulting discussion highlights what we feel were our important lessons learned, as well as some of our thoughts on how to approach collaborations or deployments with external stakeholders in sustainable computing projects.
\end{abstract}

Keywords - Sustainability, stakeholders, reflection, case study, sustainable interaction design, network services, domestic energy, data demand, policymakers, decision support

\section{Introduction}

How do we get people to care about the environmental dimensions of computing? What factors influence their consumer and lifestyle choices on macro and micro scales? What role(s) can weand should we - play, as researchers, in nudging peoples' behaviours towards sustainability? Or should we simply highlight how digital technologies are tied to sustainability? How successful are our research endeavours in doing so? What can we actually accomplish? These are some of the questions that we, the authors of this paper, have been grappling with and discussing for the past five years.

We are a group of early career researchers (i.e. three post doctoral researchers, and one recently appointed Lecturer) who undertake projects that directly or indirectly address social and environmental dimensions of computing and digital technologies. During our everyday research activities, we have worked with diverse stakeholders on diverse projects in diverse locations. In addition to designing and deploying digital systems (something we have all done to varying degrees), we have navigated and negotiated with public policy and policymakers, institutional 
processes, and diverse communities of practices. And across those diverse stakeholders and projects, we have found some shared tensions.

In this paper, we take a step back from the pressures of running new projects and instead choose to openly reflect on the similarities and differences between some of our previous projects and experiences. We briefly describe some of our unique research experiences-presenting them here as case studies - before drawing parallels across and highlighting lessons learned between those experiences. By doing so, we hope to share some of our experiences and some of our internal tensions, and engage the broader ICT4S community in a conversation about the challenges we face as researchers operating at the intersection of technology and sustainability. We also hope to highlight how we, as early career researchers, navigate the delicate landscape of ICT4S, with its many trade-offs and conundrums. Ultimately, we reflect on how we believe academics can encourage a range of stakeholders make decisions around this competing demands and decisions that have to be made by our project stakeholders.

\section{Background}

Sustainability is a multi-scalar problem space where ICTs can simultaneously provide solutions to some issues, be used to communicate problems and solutions to large audiences, and contribute to the growth and spread of ecological issues that "environmental sustainability" aims to address. Part of our job as ICT experts and researchers is exploring and examining these issues, finding who has the levers to influence them, and deciding which levers to focus on pulling. Although ICT4S is a relatively young and multidisciplinary community, conference, and research field, many researchers operating in the ICT4S community are already doing this engagement and outreach work (e.g. [1, 2, 3, 4]).

What we see less often, though, are opportunities for or example of scholars - especially early career scholars - openly reflecting about their research practices and projects. Few papers include reflective discussions of challenges, meaning that the opportunities for valuable knowledge transfer amongst peers and subsequent researchers are lost. This is understandable in such a young and interdisciplinary field: research in ICT4S has to report on the impact on environment sustainability, as well as describe the technological aspects with regard to design, implementation, and evaluation. All those are ongoing difficult issues by itself, and the engagement with stakeholders is an additional step that is always present, but oftentimes mentioned in passing.

Nevertheless, some works within the ICT4S community and other venues discussing the intersection of computing and sustainability provide first insights into stakeholder engagementsimplicitly or explicitly. Two of the most explicit discussions about stakeholder engagements are discussions about involved parties in smart city planning [5] and an overview of all the different actors in sustainable computing in general [6]. They shed light on the complexity of the system that influences, or is influenced by, any ICT research aiming to contribute to sustainabilityand that researchers need to be aware of those complexities. Our work continues and expands on this research by reporting on our experiences of dealing with this complexity and providing lessons learned for future researchers on how to engage with different stakeholders.

Stakeholder engagement oftentimes is about transferring knowledge, including sustainability knowledge; as such, discussions about teaching sustainability as well as students' opinions of and attitudes towards sustainability provide useful lessons as well. Previous work at ICT4S engages with this important question, reporting on the integration of sustainability into a technological teaching programme [7]. The issues encountered second typical issues in many other ICT4S projects, including our own research: sustainability is a broad topic, the specific 
aspects of sustainability that are being targeted are not always immediately clear, and the link between sustainability and computing is perceived as rather weak. One of the reasons is argued to be a different worldview of students who, despite being exposed to and interested in sustainability, struggle to connect the issue to their field of study (e.g., computer science). This different worldview is also reported on in two surveys of first year students in New Zealand $[1,2]$, echoing similar insights.

A group of stakeholders that can be highly influential therefore has the potential to lead to a real-world impact of computing on sustainability are policy makers. Previous work reports on a success story of engaging with policy makers by envisioning a design fiction scenario and, based on this project, drawing lessons for engaging with policy makers and politicians about energy and sustainability [8]. While this highlights a direct engagement of researchers with policy makers, another approach is to create technology that indirectly helps to influence policies, such as through ePetitions [9] and eParticipation [10]. This example also showcases how influencing a singular group of stakeholders (i.e. policy makers) can be approached by involving other parties (e.g. citizens, web service providers).

While in the previous example citizens can be interpreted as a mediator in the stakeholder network, other projects put them at the centre of the engagement; the most prominent example for such work is citizen science. Citizen science enables a broad audience without any specific background to not only participate in, but even shape the details of a project, and carry out the research themselves. Creating the technology together with researchers in workshops, citizens deploy probes for measuring sustainable impact in places of their choice, such as connected to web services to inform other people (e.g. [11]) or immediately visible to everyone in the form of spectacle computing (e.g. [12]).

ICT has the opportunity to tackle environmental (and social) grand challenges - but in order for this effort to be successful, we need to engage with and win over the stakeholders involved in those challenges. In this paper, we openly reflect on our own experiences in an attempt to help other researchers prepare for such stakeholder engagements, making the process hopefully more feasible and tractable in the future. We also hope that, by including questions in our reflections, attendees of the conference share some of their best practices, methods, and experiences with us, so that we can start to build a supportive, generative, and sustainable community of researchers and practitioners.

\section{Case studies}

The following case studies are meant to serve as vignettes; they outline some of our diverse experiences with stakeholder engagement by describing stakeholder-specific projects and perspectives. There is no specific order to the case studies.

\subsection{Designing Sustainable Consumer Electronics}

Technological advancements have contributed to the increasing amount of consumer electronics in everyday life, causing significant environmental issues through the obsolescence of old devices - in the form of an ever-growing amount of e-waste. There are many potential stakeholders involved in this project that are either part of the problem or solution, and as many ways to tackle the issue. Two potential directions that we investigated were to influence purchasing decisions of consumers through environmental information, drawing insights from marketing research $[13,14]$ and attempting to influence the design of consumer electronics itself $[15,16,17]$. In this case study we highlight the insights from the latter project, which evolved from an 
attempt to design sustainable consumer electronics into an investigation of transferring domain knowledge in general.

For this study, we recruited 14 product designers that were asked to apply sustainability guidelines (i.e., the Attachment Framework $[18,19]$ ) to their design process, as well as ten additional product design experts for the evaluation afterwards. The recruitment of the additional experts for the evaluation process became a necessity as assessing the result and success of the project became difficult due to the subjectivity of the quality of designs and the ambiguity of sustainable impact - two issues that have been discussed in research before (e.g., [20, 21, 22]). While the initial design task did not lead to a success in terms of transferring the sustainable design knowledge, we gained invaluable insights into the product design process, but also the particular challenges when bringing research into design practice [23].

There were several difficulties in the process that we did not anticipate. First, "designer" is an ambiguous term and even among "product designers" the specific expertise varies a lot. Expertise also plays a huge role, as more experienced designers seemed more difficult to recruit, but according to our insights as well as previous research (e.g., [24]) design novices and students are more open-minded to new methods. As a result of our study we learned that we had to investigate the design process thoroughly first before being able to incorporate the sustainable design knowledge into the design process. At the conclusion of the study, we had learned as much (if not more) about general lessons for stakeholder involvement than about the projectrelated questions themselves [25].

\subsection{Everyday Domestic Life}

Motivated by a massive amount of literature on behaviour change and eco-feedback and subsequent lack of nuanced understanding of the growing energy consumption in the home we have been working with domestic 'stakeholders' (or people) in an attempt to understand how digital technology and energy is implicated in everyday life and practices [26]. Our work looks to gather empirical observations from stakeholders and reflect on energy, technology and personal practices with these stakeholders using both qualitative data (e.g. photos, interviews) and quantitative analysis (e.g. visualised energy consumption). This engagement with domestic stakeholders has helped us better understand how practices, energy and digital technology are intertwined and configured [27], how technology is pertinent in different practices (e.g. cooking [28], thermal comfort [29], mobile contexts [30]), and how technology may be encouraging less sustainable everyday practices [31]. All of these research project required participants to reflect on usage or consumption that primarily related to energy use.

These empirical studies have provided a range of valuable insight relating to sustainability, including: the potentially unsustainable growth in energy associated with mobile device use and data demand [30,32]; the energy consumption of eco-feedback and home automation systems [33]; how larger more complex ecologies and constellations of devices are relied upon in the home [31] and how these consume more in use energy, data and embodied energy [27]; how students control their thermal comfort in more and less energy demanding ways [29]; and, the considerable variation in the $\mathrm{CO} 2$ footprint of student cooking practices [28]. As part of the dissemination place of our research we aimed to engage and shift the attitudes and practices of the Student Body at Lancaster University. With support of Green Lancaster and Lancaster University we used our new understandings of energy consumption and practices grounded in our empirical research to create four flyers (food, ICT, thermal comfort, energy in the home) with a design firm that aim to encourage less energy consumption and more forethought with regards to energy and sustainability in the home and on campus (e.g. Figure 1). With the 

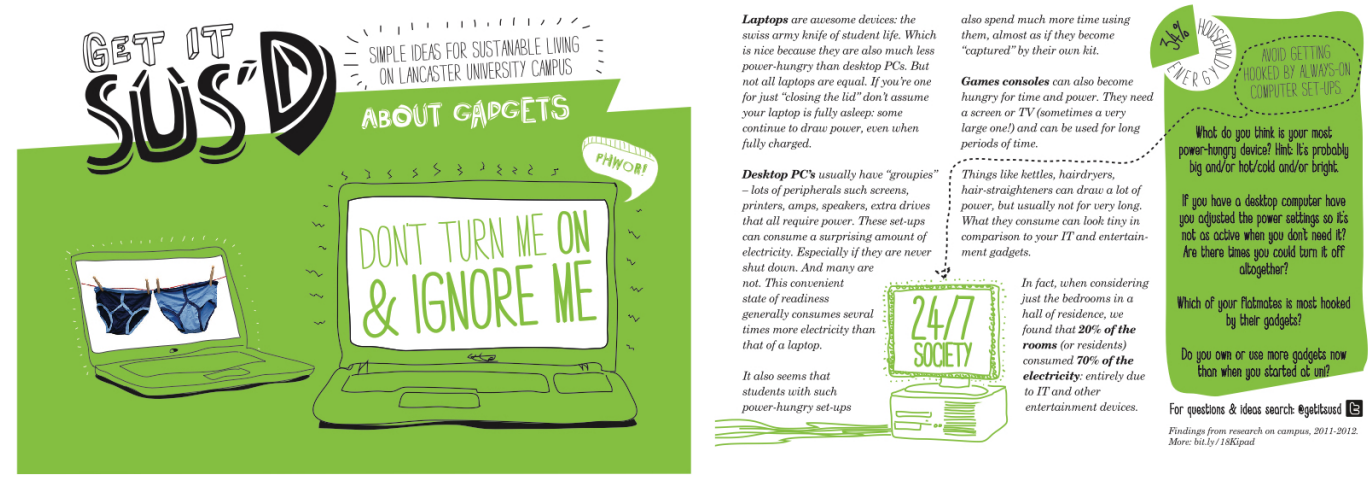

Figure 1: Don't turn me on and ignore me! Producing accessible and appropriate communications are important when engaging with stakeholders. This example was designed with students in mind.

support of the University and campus residencies the flyers were handed out to all Freshman year undergraduates during the annual "Freshers Week" fair.

\subsection{Network and Digital Infrastructure Engineers}

In the field of computer networking and networked systems, recent research has focused on highly flexible and programmable infrastructures. Originally focusing on the network itself, this is concerned with granting fine-grained control and adaption. This very much paved the way for novel functionality to be developed, replacing traditional hardware with behaviour embedded in the network. The focus of many national and international research projects, an expanse group of research organisations, universities, SMEs and large businesses have been involved in this work. Included with in this is infrastructure operators, Internet service providers and overthe-top service providers (such as video-on-demand [34] and music services). Example areas of interest from these stakeholders include caching [35, 36], quality of experience (QoE) [37], and domestic contexts [38, 39].

This flexibility in the network has recently been matched with similar flexibility in other components within digital infrastructure. Although cloud computing is a relatively mature concept, there have been recent attempts to integrate further, more disparate, resources into the infrastructure. Moving firmly away (but not discounting) the facilities offered by a wellconnected and maintained datacentre, these efforts are attempting to opportunistically leverage resources closer to the network edge, in an attempt to deliver services closer to the user. Coupled with the resources that may be available in-between these two extremes, interested parties are able to deploy services in various locations within the network. Driven by a reduction in latency and a lower probability of packet loss, this furthers the quality of experience received by their customers.

Considering the wide-reaching scope of digital infrastructure and the prevalence of the Internet, it is perhaps unsurprising to see that there are many parties involved. From the technicians responsible for running the infrastructure day-to-day, to the network architects and capacity planners who attempt to predict future growth so that long-lived hardware deployments are satisfactory for years to come. When you add into the mix the influence that regulatory and 
standardisation bodies have in this area, it becomes a long list of complex, changing relationships. The progress of technology advancements, particularly in recent years, has done nothing to simplify the matter either.

Driven by personal correspondence, conversation and general community sentiment, it is perhaps surprising to see that sustainability is often a somewhat peripheral driver to the decisions that they make, both large and small. Cost, and the reduction of such, is seemingly of a much higher priority. This is also mirrored in the academic research space, with similar reasons are often cited as motivation for work and areas of interest. And yet, the technology is becoming mature enough for all parties to benefit from these advances. This includes extending product life cycles and the ability to scale resource consumption according to demand; aspects that undoubtedly can be used to further sustainable goals in this space.

\subsection{Academics}

For just under two years, one of the authors of this paper studied the social practices of a group of computing academics, and attempted to learn about how their practices influenced the environmental footprint of computing. She did so for a few reasons. First and foremost: the environmental footprint of digital devices and services is growing at an alarming rate [40], and she wanted to understand how a group of academics who influence the design and deployment of major technologies conceived of and negotiated the environmental dimensions of their work -if they did at all. Secondly, through her position as an early career researcher, she thought that she might have easy access to a community of computing academics, and she wanted to take advantage of that access to study an understudied community's practices. Lastly, she wanted to learn about and apply social practice theory within a research setting.

Her study was entirely qualitative; she interviewed twenty-two diverse members of this academic community in their workplaces, at conferences, in local eateries, or in whatever meeting room we could find. She conducted some of her interviews in-person and others via Skype, so that she could access and attempt to analyse the practices of academics working in settings that were not immediately accessible to her. She also immersed herself in some of the academic communitys activities by attending and running workshops, assisting with course delivery, and submitting co-authored papers for peer-review. As of a result of her process, she dedicated a chapter of her doctoral thesis to describing how this specific community of computing academics directly and indirectly does and does not influence the environmental footprint of computing. She will submit this work to a journal for peer-review in the coming months.

During her research, she - perhaps unsurprisingly - found that her participants were primarily motivated by what their careers required them to be motivated to pursue: publications in high-quality venues, teaching reviews that met high-quality standards, prestigious grants, and projects that pushed the boundaries of some dimension of computing. Career stability and career progression are directly influenced by these latter factors, so many scholars - especially early career scholars - are directly motivated to gain recognition for their successful completion of the aforementioned activities. The exact shape and nature of those activities varied considerably from country to country and career-stage to career-stage (i.e. the pressures faced by a $\mathrm{PhD}$ student are not the same as a long-tenured professor).

For most of her participants, sustainability and other notions related to the environmental footprint of digital technologies played a mostly negligible role in their practices. Academic institutions and funding bodies did not prioritise or incentivise environmentally sustainable projects, so many scholars did not see a value in them. That said, several of her interviewees expressed an interest in sustainability - amongst them, what they meant by sustainability var- 
ied considerably. Some referred to environmental effects of computing, whereas others referred only to the long-term maintainability of software code (i.e. through the creation of thorough documentation, backups, and interoperability). And several established academics within this computing community had established their careers by pushing for sustainable design principles and practices. However, overall, sustainability seemed like a niche interest amongst this community of computing academics.

\subsection{Institutional Policy and Decision Makers}

Since 2015, on-going work with Lancaster University's Energy Manager and Institute "Energy Lancaster" has aimed to develop a Energy Information System (EIS) in support of the University's "Living Laboratroy" [41] for research, teaching and sustainable decision making on campus. We have designed and deployed a system utilising existing IoT and digital systems to put together a central store of all data related to buildings and energy on campus. Through a combination of action research [42] and participatory design we have worked closely with the University's Campus Manager (CM), Building Manager (BM) and Energy Manager (EM) to develop a number of user stories to help us shape the develop interfaces to EIS that promote policies and decisions promoting environmental sustainability at a campus level. These stakeholders were engaged throughout the design process.

The second group of policy and decision makers are working as part of the Freight Traffic Control project (FTC) focusing on how people and digital technology can help lower the carbon footprint, congestion and air quality issues related to the growth in parcel deliveries in Central London [43, 44]. The entire project has been created with policy and decision makers (e.g. Transport for London, TNT, Gnewt Cargo) involvement at the centre. This work sits somewhere between participatory design and co-creation with stakeholders being heavily involved in the the initial grant proposal, creation of datasets utilised by the project, the analysis of data and results, designing of new interactive visualisations, and are who a number of the public policy evidence submissions are being targeted.

Both the EIS and FTC stakeholders are motivated by mounting societal and institutional pressures to reduce carbon emissions and better utilise infrastructure. Ultimately the institutions are all motivated to do this due to the financial benefits, for EIS the project allows better use of energy and building infrastructure on campus resulting in both energy and financial saving, and for FTC the stakeholders are invested in changing the system due to marginal profits, parking fines, congestion and an inefficient use of the road infrastructure. In part due to the high motivation of these stakeholders to meet institutional and policy targets these stakeholders are all engaged with the projects, yielding a strong collaboration that in our experience has significantly contributed to the success of these projects, especially where there is clear benefits for the stakeholders.

A clear synergy between researchers and institutional policy and decision makers is where researchers fill particular skill or knowledge gaps. For example, in both cases of EIS and FTC large amount of data is collected in both socio-technical systems that has been left to gather dust due to the stakeholders having limited skills, time and funding to do anything worthwhile. If such synergies exist, partnering with institutional policy and decision makers can be a key place to have influence and propose more radical trajectories in projects that might require larger reconfiguration of infrastructures and industry.

Difficulties arise when financial backing is limited or removed, when projects have to rely on slow to move or un-invested third parties and processes (bureaucracy), when there are conflicting ideas and ideals about how to proceed in a project especially when working with 
more senior stakeholders, and when stakeholders vision goes beyond the scope or length of the project.

\section{Reflection and Discussion}

Our individual case studies highlight a variety of different experiences from our stakeholder engagements. As every author shared their stories and experiences among the other co-authors of this paper (both in writing as well as in personal conversation) we noticed patterns that were common across all projects, but also differences that emphasise unique challenges for certain collaborations. We started compiling a list of insights that we believed would have been helpful to have had at the beginning of our journey, and through discussion and writing iterated on those insights. The key takeaways in this section highlight the synthesised results of those discussions.

As we explain at the end of this section, we have opted to do this out of an interest in building a supportive, generative, and sustainable community of ICT4S researchers and practitioners. We kept the insights more open-ended rather than conceiving a checklist or framework; rather, we consider this a starting point for the community to engage in a discussion of those issuessimilar to the article by Anokwa et al. [45] for the field of HCI4D.

\subsection{Sustainability doesn't always align}

All of us - the authors - are enthusiastic about sustainability (e.g. environmental and social justice, resource depletion, design, sustainable policies, sustainable innovation) and perceive it as the primary goal, or at least one of the primary goals, in our research. However, sometimes stakeholders and practitioners have different priorities with regards to motivations and end goals. Even if they share an enthusiasm for sustainability and want to see it as a primary goal, we found that they were and are often forced in practice to let it fade into the background of their projects. Examples are reliability of the network infrastructure, speed of delivery for logistics, aesthetics in design, or pressures related to policy development [8]; all of those are still the primary concern for our stakeholders. This is also true for the computing researchers one of our authors worked with; they often saw usability and usefulness of technology as more important than sustainability.

When discussing these issues amongst ourselves, we - the authors - realised that, for us, this meant it was imperative that we learn to quickly become aware of the different priorities of our stakeholders. We need to take these differing priorities into account when we engage, establish collaborations, or prepare activities with any people or communities who might become our research stakeholders. If we fail to do this, our projects might be jeopardised from the start because practitioners are often unable to pursue sustainability goals when those goals are at odds with their everyday practice. As such, adapting to our stakeholders' specific priorities and requirements will sometimes mean that sustainability has to take the backseat. And we need to get to a point where we are comfortable with that. Setting clear goals that are suitable for a project's unique stakeholders can tremendously increase the acceptance and success rate of that project. What we can do as ICT4S researchers is add sustainability into the process such that the project's primary and stakeholder-specific goals are reached in tandem with addressing issues of sustainability. This can be especially critical for stakeholders who do not share the same enthusiasm for sustainability - some of whom might prefer that we avoid using the word sustainability as much as possible over the course of the project. 


\subsection{Identifying and engaging with stakeholders}

In our research, we found that the most important first step we could take whilst engaging with stakeholders was to identify the right group of stakeholders. Although this sounds trivial, we discussed how this actually needs a lot of careful planning, which we aren't always trained to do during our methods courses, or given the space to do if we're feeling rushed to meet a short-term paper or project deadline. But important questions about identifying stakeholders need to be asked at the outset of any projects, including: who exactly are and aren't the stakeholders that are most relevant to our project, and why? How could we approach the stakeholders and establish the right project objectives? What could we do if they weren't interested or available? How would their lack of availability — or lack of interest in our project-influence our findings or our impact on sustainability?

For example, in the project seeking to improve the longevity of networks and digital infrastructures, there were many different parties involved, such as engineers, policymakers, service providers, and so on. Each of those stakeholder groups played an important role in identifying solutions for sustainability, but determining which group to contact first depended on what the specific goals and intended approaches to the project were. Securing or failing to secure all of their buy-in or participation in the project would have also affected the overall outcomes of the project. Inappropriately, too narrowly, or too broadly selecting which of those groups of stakeholders to approach would have jeopardised the project entirely. A similar issue arose in the project with designers; oftentimes the term designers is used in research without distinguishing further if the target audience is product designers, graphic designers, or interaction designers - all of which have different work processes that require different approaches in collaborative projects [23]. Therefore, identifying the correct, narrowly defined target audience is an important, easy-to-overlook first step in any research that involves stakeholder involvement [46].

Of course, once we've identified the correct - or the available - stakeholders, the next step we have to undertake is equally challenging: we need to develop a concrete plan about how to best engage with those stakeholders. In our experiences, establishing our connections early by setting up a mutually agreeable collaboration was crucial. As many researchers know, and as we experienced [47], recruitment can be a difficult endeavour, especially in large-scale deployments. Moreover, with recruitment strategies such as snowball sampling or convenience sampling, an ill-timed or poorly phrased first contact can lead to high rejection rates and stall a research project in its very early stages. One of our researchers encountered that issue in a project not described here, and was unable to secure the quality or quantity of interviews they had wanted for their project.

As we, the authors, discussed these issues, we realised that careful planning of the recruitment process - going beyond just familiarising ourselves with recruitment methods, and instead focusing on familiarising ourselves with the work processes, practices, and interests of our target stakeholders - was critical. We needed to be able to getting our stakeholders excited about the possibilities of collaborating with us so that we could mitigate potential obstacles to collaboration. This effective dimension of recruitment was rarely, if ever, communicated to us during our research methods training, but it is absolutely critical, especially in projects where target stakeholders are a niche community. For example, in the case of policymakers, who often hold unique positions and cannot be replaced if they reject an invitation, or in the case of experienced designers who are more difficult to recruit than design students. 


\subsection{Language, communication, and revisiting our assumptions}

At this point - and as multidisciplinary communities like ICT4S already know -it's fairly banal to say that different disciplines and genres of work have unique and specific terminology. We already know that this terminology often helps to simplify and expedite communication amongst expert practitioners. However, for people who are not affiliated with a field's niche terms, meanings, and language, it can be a serious obstacle for communication during a project. As we, the authors, chatted amongst ourselves about these issues, we realised that, for us, it was oftentimes incredibly difficult to notice when we had erected such language barriers. Some of our discipline-specific terminology had become so commonplace to us that we struggled to identify which terms were and were not specialised in our discipline.

What the meant for us was that we needed to take extra steps towards being reflexive and reflective about what language we used when we worked with our stakeholders [47]. We agreed that it was especially important to familiarise ourselves with our stakeholders' specific language, too, so that we could apply it when working within the project [46]. This also meant learning what terms we shouldn't use when talking with our stakeholders. For example, in a few cases, we realised that our stakeholders were averse to the term "sustainability", so we opted to use the term resilience or not use language related to sustainability at all.

In the projects that involved engaging with policymakers, we realised that not only the language itself, but also the way that our message was delivered played an important role. To echo Marshall McLuhan, "the medium is the message" [48]. While policymakers do value examples of scientific research and an overview of the available knowledge, we found that supporting those insights by stories is a promising way to increase understanding and engagement [8]. Stories contextualise data and make it tangible, allowing audiences to visualise the situation and relate to the scientific insights more directly.

Similarly, when trying to engage a larger volume of stakeholders and have a larger impact, dissemination beyond academic papers and industry reports are important. For example, if based on your research you understand that to have a $10 \%$ reduction in energy consumption on an University Campus you have to change an entire student bodies' attitude towards cooking, heating and ICT use you need a) to be able to reach them, and b) have long term institutional backing to ensure engagement with the target audience. Design patterns - as initially envisioned by Alexander [49] - offer another way of translating research knowledge into artefacts that can be used by non-experts.

Learning these lessons weren't easy for us. Few of us felt like we had been adequately trained - during our predominantly technical education - in how to navigate and negotiate these sorts of socio-linguistic issues. As we discussed the challenges we faced during our projects, we were left wondering: what more can be done to prepare early career researchers for these communication issues? How can we help our peers to become aware of the community-specific language we're using and how it might impact a project? Where do most academics discuss these issues? They do not appear to be present in many publications.

\subsection{How we define sustainability matters}

Sustainability is the common motivating theme among all our case studies, and the overarching goal we as researchers are all aiming for. However, as we experienced in our projects, sustainability is also a broad term that needs to be defined and specified for each project individually. For example, in the design study our goal was to address obsolescence through the design of technology, increasing the longevity of devices. In the case study about parcel deliveries and campus energy, the goals of the researchers and the stakeholders aligned, mo- 
tivated to reduce pollution, use resources more responsibility and lower carbon emissions by transforming the ways infrastructures are provisioned and used. Motivated by financial costs, the network and digital infrastructure stakeholders are concerned with: an increased lifespan of the infrastructure, but also higher energy efficiency or reduced consumption. Each of those examples contribute to sustainability, but in entirely different ways; as others have mentioned before, sustainability is a broad term that can be approached on various levels [50] but needs to be specific on a per-project basis [51]. For us, one of the pitfalls that we encountered related to ensuring that sustainability referred to environmental sustainability - as mentioned in the case study about computing academics, in some settings, sustainability carries a different meaning related to the long-term maintenance of software.

\subsection{Ripples, rebounds and the Hawthorne Effect}

In our projects, especially in stakeholder engagements that are long-term collaborations, we found that it was interesting to observe how attitudes towards sustainability changed over time; especially, comparing the stakeholders view on sustainability before and after the project. While this can be one approach to engage with the difficult task of evaluating research [52, 53], it also exposes the project to other issues. For example, by observing a population--and especially actively engaging with it-researchers inevitably provoke change, commonly known as the Hawthorne Effect. From the ethical point of view of us being interested in promoting sustainability it might not be a significant issue as the change most likely results in a more sustainable outcome; however, it limits the insights gained as well as the generalisability of research. As with so many side effects triggered by research (cf. Jevons Paradox and Rebound Effects $[54,55])$ it is difficult if not near impossible to counter those, but it is important to acknowledge and minimise them.

A related ethical issue that is already well-known in research areas such as development and health is that of the long-term effects of technology interventions provided to a population and the potential removal thereof. For example, in the design study we deployed prototypes that helped designers to create more sustainable products, but what are the effects of removing such prototypes? Few academic research projects seem able to sustain long-term deployments. As we discussed this amongst ourselves, we agreed that we felt like projects should have a long-term plan beyond the deployment and collaboration phase how to maintain the post-project state, especially when a project has goals related to changing peoples behaviour. But we also felt like this was a difficult challenge for academics to actually address, especially if no funding or formal support existed after a project ended. Who should be responsible for maintaining projects at the official end of them? How, when, and for what purposes can that responsibility be shared? Is there a way to restructure the responsibilities that academics and academic institutions have to the communities and stakeholders with whom they work? We don't have answers to these questions, nor were we prepared for them in our early career research training. But they feel important, especially for any future work related to broad notions of social and environmental sustainability.

\subsection{The stakeholders that research forgot}

Through our case studies we've attempted to demonstrate how interactions and research with stakeholders can a) reveal useful insights into how to engage with stakeholders about sustainability, and b) demonstrated how sustainability projects can speak to a variety of stakeholders (e.g. designers, domestic stakeholders, network infrastructure engineers, academics, industry and policy makers). Whilst all of the case studies presented provide evidence of how to work 
with a variety of stakeholders, they all lack awareness of how effecting change with these particular stakeholder groups can have unconsidered and profound effects on the lives of stakeholders that are not considered. For example, any change in institutional policy at a university may effect the academics, students, and people working and living on campus ([41]) and the people and citizens whose lives will be affected the most by decisions made for them by institutional stakeholders $([47])$.

\subsection{Building a supportive, generative, and sustainable ICT4S com- munity}

We wanted to bring this set of case studies, reflections, and questions to ICT4S to spark a conversation - at a ConverStation - amongst our fellow practitioners, in part because we are firm believes in the value of open reflection ([56]). But also because we have grown to believe that these conversations need to have a place and a space in our community. We want to know: how do others deal with the issues we've raised? Where and how can we reflect and share these lessons in our literature? What more can we do to support early career researchers who are struggling to work within this complex socially, politically, spatially, environmentally, and at-times personally demanding research community? We hope that attendees of the conference can share some of their best practices, methods, and experiences with us, so that we can start to build a supportive, generative, and sustainable community of ICT4S researchers and practitioners.

\section{Conclusion}

In this paper, we shared stories from our research projects with different stakeholder engagements and the insights we gained from those collaborations and deployments. Engaging with audiences outside of the field, in particular in our research that aims to tackle issues of sustainability, is a challenging task - environmental issues are often too complex to be dealt with in isolation, making the involvement of stakeholders an important step. In reflecting on our individual case studies and comparing our insights we derived lessons learned that we hope will empower future researchers, in particular early career researchers who are at the beginning of their projects, to take up those challenges more effectively. In addition, we hope that our discussion will enrich the already existing debate about stakeholder engagement within the ICT4S community and beyond to increase the impact of computing on sustainability.

\section{Acknowledgment}

The authors would like to thank Kelly Widdicks for her support and input on early discussions about this paper. Portions of this work was performed as part of an EPSRC-funded project (EP/N02222X/1) called Freight Traffic Control 2050 (http://www.ftc2050.com/). We also thank Energy Lancaster of Lancaster University for their continued support of the development of the Energy Information System and Sustainable Living Laboratory. 


\section{References}

[1] S. Mann, M. Lopez, D. Lopez, and N. Smith, Educating for ICT4S: Unpacking sustainability and ethics of ICT student intakes. Atlantis Press, Aug 2015. [Online]. Available: /proceedings/ict4s-env-15/25836171

[2] S. Mann, K. Costello, M. Lopez, D. Lopez, and N. Smith, An ethical basis for sustainability in the worldviews of first year students. Atlantis Press, Jul 2014. [Online]. Available: /proceedings/ict4s-14/13433

[3] J. Townsend, "Web for sustainability: Tackling environmental complexity with scale," in ICT for Sustainability 2014 (ICT4S-14), Stockholm, Sweden, August 25, 2014., 2014. [Online]. Available: https://doi.org/10.2991/ict4s-14.2014.8

[4] - "Digital taxonomy for sustainability," in ICT for Sustainability 2015 (ICT4S-15), Copenhagen, Denmark, July 2015., 2015. [Online]. Available: https://doi.org/10.2991/ ict4s-env-15.2015.33

[5] A. Kramers, M. Hjer, and J. Wangel, Planning for smart sustainable cities: Decisions in the planning process and actor networks. Atlantis Press, Jul 2014. [Online]. Available: /proceedings/ict4s-14/13454

[6] C. Herzog, L. Lefèvre, and J.-M. Pierson, Actors for Innovation in Green IT. Cham: Springer International Publishing, 2015, pp. 49-67. [Online]. Available: https://doi.org/10.1007/ 978-3-319-09228-7_3

[7] E. Eriksson and D. Pargman, ICT4S Reaching Out: Making sustainability relevant in higher education. Atlantis Press, Jul 2014. [Online]. Available: /proceedings/ict4s-14/13423

[8] V. Thomas, C. Remy, M. Hazas, and O. Bates, "Hci and environmental public policy: Opportunities for engagement," in Proceedings of the 2017 CHI Conference on Human Factors in Computing Systems, ser. CHI '17. New York, NY, USA: ACM, 2017, pp. 6986-6992. [Online]. Available: http://doi.acm.org/10.1145/3025453.3025579

[9] S. McDonald, B. Nardi, and B. Tomlinson, "Political realities of digital communication: The limits of value from digital messages to members of the us congress," in Proceedings of the 2017 Workshop on Computing Within Limits, ser. LIMITS '17. New York, NY, USA: ACM, 2017, pp. 129-138. [Online]. Available: http://doi.acm.org/10.1145/3080556.3080565

[10] U. Wehn and J. Evers, Citizen observatories of water: Social innovation via eParticipation. Atlantis Press, Jul 2014. [Online]. Available: /proceedings/ict4s-14/13419

[11] S. Kuznetsov, G. Davis, J. Cheung, and E. Paulos, Ceci NEst Pas Une Pipe Bombe: Authoring Urban Landscapes with Air Quality Sensors, ser. CHI 11. ACM, 2011, p. 23752384. [Online]. Available: http://doi.acm.org/10.1145/1978942.1979290

[12] S. Kuznetsov, G. N. Davis, E. Paulos, M. D. Gross, and J. C. Cheung, Red balloon, green balloon, sensors in the sky, ser. UbiComp 11. ACM, 2011, p. 237246. [Online]. Available: http://doi.acm.org/10.1145/2030112.2030145

[13] C. Remy and E. M. Huang, The complexity of information for sustainable choices, ser. CHI 12 workshop, 2012.

[14] C. Remy, "Taking a note from marketing research in sustainable hci," interactions, vol. 20, no. 3, May 2012.

[15] C. Remy and E. M. Huang, Tailoring Sustainable HCI Design Knowledge to Design Practice, ser. CHI 14 workshop, 2014.

[16] - Addressing the Obsolescence of End-User Devices: Approaches from the Field of Sustainable $H C I$, ser. Advances in Intelligent Systems and Computing. Springer International Publishing, 2015, p. 474.

[17] —, "Limits and sustainable interaction design: Obsolescence in a future of collapse and resource scarcity," First Monday, vol. 20, no. 8, Jul 2015. [Online]. Available: http://firstmonday.org/ojs/index.php/fm/article/view/6122 
[18] W. Odom, J. Pierce, E. Stolterman, and E. Blevis, Understanding why we preserve some things and discard others in the context of interaction design, ser. CHI 09. ACM, 2009, p. 10531062. [Online]. Available: http://doi.acm.org/10.1145/1518701.1518862

[19] S. Gegenbauer and E. M. Huang, Inspiring the design of longer-lived electronics through an understanding of personal attachment, ser. DIS 12. ACM, 2012, p. 635644. [Online]. Available: http://doi.acm.org/10.1145/2317956.2318052

[20] A. Salovaara, A. Oulasvirta, and G. Jacucci, Evaluation of Prototypes and the Problem of Possible Futures, ser. CHI 17. ACM, 2017, p. 20642077. [Online]. Available: http: //doi.acm.org/10.1145/3025453.3025658

[21] M. S. Silberman and B. Tomlinson, Toward an ecological sensibility: tools for evaluating sustainable HCI, ser. CHI EA 10. ACM, 2010, p. 34693474. [Online]. Available: http://doi.acm.org/10.1145/1753846.1754003

[22] A. Dix, Theoretical analysis and theory creation. Cambridge University Press, 2008.

[23] C. Remy, S. Gegenbauer, and E. M. Huang, Bridging the Theory-Practice Gap: Lessons and Challenges of Applying the Attachment Framework for Sustainable HCI Design, ser. CHI 15. ACM, 2015, p. 13051314. [Online]. Available: http://doi.acm.org/10.1145/2702123.2702567

[24] N. Cross, "Expertise in design: an overview," Design Studies, vol. 25, no. 5, p. 427441, Sep 2004.

[25] C. Remy, "Incorporating sustainable hci research into design practice," Ph.D. dissertation, University of Zurich, 2017.

[26] O. Bates, A. K. Clear, A. Friday, M. Hazas, and J. Morley, "Accounting for energy-reliant services within everyday life at home," in Proceedings of the 10th international conference on Pervasive Computing, ser. Pervasive'12. Berlin, Heidelberg: Springer-Verlag, 2012, pp. 107-124. [Online]. Available: http://dx.doi.org/10.1007/978-3-642-31205-2_8

[27] O. Bates, M. Hazas, A. Friday, J. Morley, and A. K. Clear, "Towards an holistic view of the energy and environmental impacts of domestic media and it," in Proceedings of the SIGCHI Conference on Human Factors in Computing Systems, ser. CHI '14. New York, NY, USA: ACM, 2014, pp. 1173-1182. [Online]. Available: http://doi.acm.org/10.1145/2556288.2556968

[28] A. K. Clear, M. Hazas, J. Morley, A. Friday, and O. Bates, "Domestic food and sustainable design: A study of university student cooking and its impacts," in Proceedings of the SIGCHI Conference on Human Factors in Computing Systems, ser. CHI '13. New York, NY, USA: ACM, 2013, pp. 2447-2456.

[29] A. K. Clear, J. Morley, M. Hazas, A. Friday, and O. Bates, "Understanding adaptive thermal comfort: new directions for UbiComp," in Proc. of UbiComp, 2013, pp. 113-122. [Online]. Available: http://doi.acm.org/10.1145/2493432.2493451

[30] K. Widdicks, O. Bates, M. Hazas, A. Friday, and A. R. Beresford, "Demand around the clock: Time use and data demand of mobile devices in everyday life," in Proceedings of the 2017 CHI Conference on Human Factors in Computing Systems, ser. CHI '17. New York, NY, USA: ACM, 2017, pp. 5361-5372. [Online]. Available: http://doi.acm.org/10.1145/3025453.3025730

[31] O. Bates, C. Lord, B. Knowles, A. K. Clear, M. Hazas, and A. Friday, "Exploring (un)sustainable growth of digital technologies in the home," in Proc of ICT for Sustainability, 2015.

[32] C. Lord, M. Hazas, A. K. Clear, O. Bates, R. Whittam, J. Morley, and A. Friday, "Demand in my pocket: mobile devices and the data connectivity marshalled in support of everyday practice," in Proceedings of the 33rd Annual ACM Conference on Human Factors in Computing Systems. ACM, 2015, pp. 2729-2738.

[33] O. Bates and M. Hazas, "Exploring the hidden impacts of homesys: Energy and emissions of home sensing and automation," in Proceedings of the 2013 ACM Conference on Pervasive and Ubiquitous Computing Adjunct Publication, ser. UbiComp '13 Adjunct. New York, NY, USA: ACM, 2013, pp. 809-814. [Online]. Available: http://doi.acm.org/10.1145/2494091.2497322

[34] M. Mu, M. Broadbent, A. Farshad, N. Hart, D. Hutchison, Q. Ni, and N. Race, "A scalable user fairness model for adaptive video streaming over sdn-assisted future networks," IEEE Journal on 
Selected Areas in Communications, vol. 34, no. 8, pp. 2168-2184, 2016.

[35] M. Broadbent, P. Georgopoulos, V. Kotronis, B. Plattner, and N. Race, "Opencache: Leveraging sdn to demonstrate a customisable and configurable cache," in Computer Communications Workshops (INFOCOM WKSHPS), 2014 IEEE Conference on. IEEE, 2014, pp. 151-152.

[36] P. Georgopoulos, M. Broadbent, B. Plattner, and N. Race, "Cache as a service: Leveraging sdn to efficiently and transparently support video-on-demand on the last mile," in Computer Communication and Networks (ICCCN), 2014 23rd International Conference on. IEEE, 2014, pp. $1-9$.

[37] P. Georgopoulos, Y. Elkhatib, M. Broadbent, M. Mu, and N. Race, "Towards network-wide qoe fairness using openflow-assisted adaptive video streaming," in Proceedings of the 2013 ACM SIGCOMM workshop on Future human-centric multimedia networking. ACM, 2013, pp. 15-20.

[38] O. Bates and M. Broadbent, "Homeflow: inferring device usage with network traces," in Proceedings of the 2013 ACM conference on Pervasive and ubiquitous computing adjunct publication. ACM, 2013, pp. 815-820.

[39] T. Fratczak, M. Broadbent, P. Georgopoulos, and N. Race, "Homevisor: Adapting home network environments," in Software Defined Networks (EWSDN), 2013 Second European Workshop on. IEEE, 2013, pp. 32-37.

[40] M. Hazas, J. Morley, O. Bates, and A. Friday, "Are there limits to growth in data traffic?: On time use, data generation and speed," in Proceedings of the Second Workshop on Computing Within Limits, ser. LIMITS '16. New York, NY, USA: ACM, 2016, pp. 14:1-14:5. [Online]. Available: http://doi.acm.org/10.1145/2926676.2926690

[41] O. Bates and A. Friday, "Beyond data in the smart city: Repurposing existing campus iot," IEEE Pervasive Computing, vol. 16, no. 2, pp. 54-60, April 2017.

[42] G. R. Hayes, "The relationship of action research to human-computer interaction," ACM Trans. Comput.-Hum. Interact., vol. 18, no. 3, p. 15:115:20, Aug 2011.

[43] O. Bates, B. Knowles, and A. Friday, "Are people the key to enabling collaborative smart logistics?" in Proceedings of the 2017 CHI Conference Extended Abstracts on Human Factors in Computing Systems, ser. CHI EA '17. New York, NY, USA: ACM, 2017, pp. 1494-1499. [Online]. Available: http://doi.acm.org/10.1145/3027063.3053128

[44] J. Allen, M. Piecyk, M. Piotrowska, F. McLeod, T. Cherrett, K. Ghali, T. Nguyen, T. Bektas, O. Bates, A. Friday, S. Wise, and M. Austwick, "Understanding the impact of e-commerce on last-mile light goods vehicle activity in urban areas: The case of london," Transportation Research Part D: Transport and Environment, 2017. [Online]. Available: http://www.sciencedirect.com/science/article/pii/S136192091730305X

[45] Y. Anokwa, T. N. Smyth, D. Ramachandran, J. Sherwani, Y. Schwartzman, R. Luk, M. Ho, N. Moraveji, and B. DeRenzi, "Stories from the field: Reflections on hci4d experiences," Information Technologies \& International Development, vol. 5, no. 4, p. 101116, Dec 2009.

[46] C. Remy and E. M. Huang, Communicating SHCI Research to Practitioners and Stakeholders. Routledge, 2017.

[47] V. Thomas, D. Wang, L. Mullagh, and N. Dunn, "Wheres wally? in search of citizen perspectives on the smart city," Sustainability, vol. 8, no. 3, p. 207, 2016.

[48] M. MacLuhan, The Medium is the Message, ser. Marshall McLuhan unbound project. Gingko Press, 2005. [Online]. Available: https://books.google.dk/books?id=NwUMuAAACAAJ

[49] C. Alexander, S. Ishikawa, and M. Silverstein, A Pattern Language: Towns, Buildings, Construction. Oxford University Press, 1977.

[50] O. Bates, V. Thomas, and C. Remy, "Doing good in hci: Can we broaden our agenda?" interactions, vol. 24, no. 5, pp. 80-82, Aug. 2017. [Online]. Available: http://doi.acm.org/10.1145/3121386

[51] M. S. Silberman, L. Nathan, B. Knowles, R. Bendor, A. Clear, M. Hkansson, T. Dillahunt, and J. Mankoff, "Next steps for sustainable hci," interactions, vol. 21, no. 5, p. 6669, Sep 2014. 
[52] C. Remy, O. Bates, V. Thomas, and E. M. Huang, The Limits of Evaluating Sustainability. ACM Press, Jun 2017.

[53] C. Remy, O. Bates, A. Dix, V. Thomas, M. Hazas, A. Friday, and E. M. Huang, Evaluation beyond Usability: Validating Sustainable HCI Research, ser. CHI 18, 2018.

[54] S. J. Kaufman and M. S. Silberman, Rebound effects in sustainable HCI, ser. CHI 11 workshop, 2011.

[55] L. M. Hilty, Information Technology and Sustainability. Books On Demand, Jun 2008.

[56] M. Brueggemann, A. Strohmayer, M. Marshall, N. Birbeck, and V. Thomas, "Reflexive practices for the future of design education: An exercise in ethno-empathy," The Design Journal, vol. 20, no. 1, pp. 1260-1269, 2017. 